\title{
Pressure-induced changes in the structural and magnetic properties of $\mathrm{YFe}_{2} \mathrm{D}_{4.2}$
}

\author{
O. Isnard,,$^{1, *}$ V. Paul-Boncour, ${ }^{2}$ Z. Arnold, ${ }^{3}$ C. V. Colin, ${ }^{1}$ T. Leblond, ${ }^{2}$ J. Kamarad, ${ }^{3}$ and H. Sugiura ${ }^{4}$ \\ ${ }^{1}$ Institut Néel, CNRS/Université Joseph Fourier, B.P. 166, 38042 Grenoble, France \\ ${ }^{2}$ Institut de Chimie et des Matériaux de Paris Est, CMTR, CNRS and UPEC, 2-8 rue H. Dunant, 94320 Thiais Cedex, France \\ ${ }^{3}$ Institute of Physics AS CR v.v.i., Na Slovance 2, 18221 Prague 8, Czech Republic \\ ${ }^{4}$ Gradual School of Integrated Science, Yokohama City University, 22-2 Seto, Kanazawa-ku, Yokohama 236-0027, Japan
}

(Received 1 March 2011; revised manuscript received 24 July 2011; published 20 September 2011)

\begin{abstract}
The study of the structural and magnetic properties of $\mathrm{YFe}_{2} \mathrm{D}_{4.2}$ under high pressure has been performed by combining energy dispersive $\mathrm{x}$-ray diffraction at room temperature up to $5.5 \mathrm{GPa}$, neutron diffraction studies at low temperature up to $2.5 \mathrm{GPa}$, and magnetization measurements up to $1 \mathrm{GPa}$. The monoclinic structure of $\mathrm{YFe}_{2} \mathrm{D}_{4.2}$ at ambient temperature and pressure is related to a distortion of the initial cubic $\mathrm{C} 15$ structure of $\mathrm{YFe}_{2}$ by the ordering of inserted D atoms. A structural transition from monoclinic to cubic structure is observed at $4 \mathrm{GPa}$. The high pressure cubic $\mathrm{YFe}_{2} \mathrm{D}_{4.2}$ has a $19 \%$ higher cell volume than that of $\mathrm{YFe}_{2}$. The application of an external pressure leads to the progressive decrease of the transition temperature $T_{M 0}$ from ferro to antiferromagnetic order $\left(T_{M 0}=84 \mathrm{~K}\right.$ at ambient pressure) and even to the suppression of the ferromagnetic ground state for pressures larger than $0.54 \mathrm{GPa}$. Moreover using the compressibility value determined by $\mathrm{x}$-ray diffraction $(\kappa=$ $\left.0.013 \mathrm{GPa}^{-1}\right)$, the decrease of volume at the critical pressure of $0.54 \mathrm{GPa}(0.70 \%)$ is in very good agreement with the increase of volume observed at the transition from antiferromagnetic to ferromagnetic state at ambient pressure $(0.65 \%)$, indicating the crucial role of volume effect. An antiferromagnetic structure was detected between $T_{M 0}$ and $T_{N}$ and for pressures larger than $0.54 \mathrm{GPa}$ in the whole temperature range below $T_{N}$. In addition, a large pressure effect on the spontaneous magnetization $M_{S}$ for pressures below $0.4 \mathrm{GPa}, d \ln M_{S} / d P=-7.3 \times$ $10^{-2} \mathrm{GPa}^{-1}$ was discovered. This clearly proves the delocalized character of the iron $3 d$ band magnetism in $\mathrm{YFe}_{2} \mathrm{D}_{4.2}$ compound. The pressure evolution of the magnetic phase diagram of $\mathrm{YFe}_{2} \mathrm{D}_{4.2}$ has been determined.
\end{abstract}

DOI: 10.1103/PhysRevB.84.094429

PACS number(s): 64.70.-p, 61.05.cp, 75.30.Cr

\section{INTRODUCTION}

Rare earth and transition metal compounds have been extensively studied as materials for hydrogen storage for the purpose of using hydrogen as a vector of energy, but hydrogen insertion is also a way to tune the magnetic properties of these compounds. ${ }^{1}$ Hydrogen insertion leads to a cell volume increase, a lowering of the crystal symmetry, and a modification of the electronic structure, which can strongly influence the magnetic interactions. Several studies were already performed on $\mathrm{RMn}_{2}$ hydrides, showing a strong interplay between magnetic and deuterium order in such systems. ${ }^{2,3}$ Competition between ferromagnetic and antiferromagnetic interactions have been reported in Laves hydrides. ${ }^{4}$ The $\mathrm{YFe}_{2} \mathrm{D}_{x}$ system is particularly interesting since it is known to exhibit several phases depending on the deuterium content. ${ }^{5}$ Seven different crystal structures have been reported starting from the simple $\mathrm{C} 15$ cubic structure of the $\mathrm{YFe}_{2}$ intermetallic to the orthorhombic phase reported for the deuterium richer $\mathrm{YFe}_{2} \mathrm{D}_{5}$ compound. ${ }^{6,7}$ Such rich structural phase diagram arises from the ordering of deuterium atoms in the tetrahedral interstitial sites of the intermetallic, thus leading to either lattice distortion and/or superstructures. The complex crystal structure of the $\mathrm{YFe}_{2} \mathrm{D}_{4.2}$ compound has only been recently solved by Ropka et al. ${ }^{8}$ by combining synchrotron and neutron diffraction studies. They established that below $323 \mathrm{~K} \mathrm{YFe}_{2} \mathrm{D}_{4.2}$ compound crystallizes in a fully ordered, monoclinic structure described in the $P c$ space group with $a$ $=5.5066 \AA, b=11.4823 \AA, c=9.4292 \AA, \beta=122.33^{\circ}$, and $V=503.765 \AA^{3}$ at $290 \mathrm{~K}$. The relationship between this monoclinic crystal structure and the original cubic cell of the $\mathrm{YFe}_{2}$ compound is illustrated in Fig. 1.
The thermal evolution of the structure and the stability of the $\mathrm{YFe}_{2} \mathrm{D}_{4.2}$ compound has been investigated by in situ neutron diffraction and differential scanning calorimetry ${ }^{9}$ showing that the desorption process is dominated by phase transitions.

The $\mathrm{YFe}_{2} \mathrm{D}_{x}$ compounds are ferromagnets at low temperature for $x<5 .{ }^{10} \mathrm{YFe}_{2} \mathrm{D}_{5}$ displays a weak ferromagnetic behavior without any ordered Fe magnetic moment. ${ }^{6}$ The Curie temperature of the $\mathrm{YFe}_{2} \mathrm{D}_{x}$ compounds decreases from $720 \mathrm{~K}$ for $x=1.3$ to $360 \mathrm{~K}$ for $x=3.5$. The situation is more complex for intermediate concentrations $3.5<x<5$. $\mathrm{YFe}_{2} \mathrm{D}_{4.2}$ exhibits a ferromagnetic order below a temperature called $T_{M}\left[T_{M 0}(\mathrm{~B}=0)=84 \mathrm{~K}\right] .{ }^{11}$ This transition temperature is found to increase linearly with the applied field. At $T_{M}$, a sharp decrease of the magnetization occurs, which was attributed to a transition from a ferromagnetic (FM) toward an antiferromagnetic (AFM) state accompanied by a $0.5 \%$ cell volume decrease. ${ }^{12}$ This magnetic transition was explained by the itinerant electron metamagnetic (IEM) behavior of one particular $\mathrm{Fe}$ site among eight $\mathrm{Fe}$ sites of the monoclinic structure of $\mathrm{YFe}_{2} \mathrm{D}_{4.2}$. This behavior shows a resemblance to that of $\mathrm{C} 14 \mathrm{AFe}_{2}$ with nonmagnetic $A$ element $(A=\mathrm{Nb}$, Ta, Hf) in particular $\mathrm{Hf}_{1-x} \mathrm{Ta}_{x} \mathrm{Fe}_{2}(x=0.1$ and 0.2$)$ for which the magnetic ground state have been reported to be at the verge of the onset of ferromagnetism. ${ }^{13-16}$ IEM behavior has also been extensively studied in $\mathrm{RCO}_{2}$ compounds and is very sensitive to a change of volume or to an internal or external magnetic field. ${ }^{17-19}$

In $\mathrm{YFe}_{2} \mathrm{D}_{4.2}$, the $\mathrm{AFM}$ order above $T_{M 0}$ is featured by a doubling of the magnetic cell along the $b$ axis. ${ }^{12,20}$ The transition from the AFM order toward a paramagnetic state is observed at the Néel temperature $T_{N}=131 \mathrm{~K}$. In addition 




FIG. 1. (Color online) Crystal structure relation between the original cubic cell of the $\mathrm{YFe}_{2}$ compound with the rhombohedral (hexagonal setting) and monoclinic ones. For simplicity, the hydrogen atoms have been omitted. The large and small circles refer to the $\mathrm{Y}$ and $\mathrm{Fe}$ atoms, respectively.

$\mathrm{YFe}_{2} \mathrm{D}_{4.2}$ phase exhibits a large magnetic entropy variation around $T_{M}\left(-\Delta S_{M}=10.83 \mathrm{~J} \mathrm{Kg}^{-1} \mathrm{~K}^{-1}\right.$ for $\left.\Delta B=5 \mathrm{~T}\right){ }^{21}$

Last but not least, a huge isotope effect on the magnetic properties has been reported for $\mathrm{YFe}_{2}\left(\mathrm{D}_{1-x} \mathrm{H}_{x}\right)_{4.2}$ compounds. ${ }^{11,12}$ The magnetovolumic transition temperature from the FM to the AFM structure is shifted from 84 to $131 \mathrm{~K}$ between the deuteride and the hydride, which corresponds to a $50 \%$ decrease of transition temperature. Such large influence of hydrogen by deuterium substitution was never observed before on the magnetic properties of a metal hydride. This difference of transition temperature was attributed to the difference of cell volume, which is $0.83 \%$ larger for the hydride compared to the deuteride. This large isotope volume effect was explained by the difference of amplitude of zero point vibration between D and $\mathrm{H}$ atom in the condensed phase.

In order to better understand the influence of the volume change on the structural and magnetic properties of the $\mathrm{YFe}_{2} \mathrm{D}_{4.2}$ compound we have focused this article on the investigation of the external pressure effect. After a short presentation of neutron powder diffraction measurements performed at ambient pressure above room temperature, the effect of an applied pressure on the crystal unit cell is investigated by energy dispersive $\mathrm{x}$-ray diffraction up to $5 \mathrm{GPa}$. Then neutron diffraction study of the temperature and pressure dependence up to $4 \mathrm{GPa}$ of the magnetic structure is presented. The isofield thermomagnetic curves as well as magnetic isotherms studied up to $0.85 \mathrm{GPa}$ are used to determine detailed magnetic properties.

\section{EXPERIMENTAL}

The preparation and characterization of the intermetallic and the deuteride are described in Refs. 22 and 23.

Neutron diffraction measurements performed above $300 \mathrm{~K}$ have been recorded on the D1B instrument operated by the CNRS at the Institut Laue Langevin (ILL, Grenoble, France) using a vanadium heating element in order to avoid extra Bragg reflection from the furnace and below $300 \mathrm{~K}$ in a cryostat. During the neutron diffraction measurements, the powder sample was contained in a vanadium cylindrical sample container of $8 \mathrm{~mm}$ in diameter. On the D1B instrument, the diffraction patterns have been recorded over an angular range of $80^{\circ}(2 \theta)$ using a multidetector with a step of $0.2^{\circ}$ between each of the $400^{3} \mathrm{He}$ detection cells. In this configuration D1B is operating with a wavelength of $\lambda=2.52 \AA$ selected by a (002) Bragg reflection of a pyrolitic graphite monochromator, the take off angle being $44.2^{\circ}$ in $2 \theta$. Due to the high flux available on the instrument, a diffraction pattern has been recorded in situ every $2 \mathrm{~K}$.

The x-ray diffraction patterns of $\mathrm{YFe}_{2} \mathrm{D}_{4.2}$ were measured at room temperature with applied pressure up to $5 \mathrm{GPa}$ by an energy dispersive-type $\mathrm{x}$-ray diffraction system with pure $\mathrm{Ge}$ detector. The $\mathrm{x}$ ray source was a $\mathrm{W}$ tube operated at $47.5 \mathrm{kV}$ and $27.5 \mathrm{~mA}$. The energy axis of energy spectrum and slit angle in diffraction were calibrated by using fluorescence lines and diffraction lines in cubic InAs patterns.

The neutron powder diffraction patterns were collected on the G6.1 high-pressure diffractometer ${ }^{24}$ on the Orphée reactor of the Laboratoire Léon Brillouin ( LLB, Saclay, France). The zero-pressure neutron diffraction patterns have been recorded previously on the G4.1 spectrometer from LLB.

The powder was mixed with $\mathrm{NaCl}$ and introduced in highpressure sapphire anvils. The applied pressure was calibrated using fluorescence of a ruby powder added as internal standard, and the measurements were done at $0.5,1,2.6$, and $4 \mathrm{GPa}$. The high-pressure cell was introduced in an He cryostat in order to perform the neutron measurements at various temperatures between 1.5 and $180 \mathrm{~K}$ for each applied pressure. The neutron powder diffraction patterns were measured between 5 and $85^{\circ}$ with a wavelength of $4.70 \AA$.

The magnetization measurements at high hydrostatic pressure up to $1 \mathrm{GPa}$ were performed on a powder sample in a superconducting quantum interference device magnetometer (Quantum Design Co.) using a miniature piston-cylinder $\mathrm{CuBe}$ pressure cell. It was filled with a mixture of mineral oils as a pressure transmitting medium. The pressure was determined at low temperatures using the known pressure dependence of the critical temperature of the superconducting state of the $\mathrm{Pb}$ sensor placed inside the cell. ${ }^{25}$ The studies were performed at magnetic fields up to $7 \mathrm{~T}$ in the temperature range from 2 to 300 $\mathrm{K}$. The evolution of the ordering temperature under different pressures was determined from temperature dependence of the low-field magnetization measured in a magnetic field of $0.03 \mathrm{~T}$ at a heating rate of $1 \mathrm{~K}$ per minute. It was defined as inflexion point on magnetization vs temperature curves. The saturation magnetization and critical magnetic fields at different pressures were determined from the isothermal magnetization curves.

\section{DIFFRACTION RESULTS}

\section{A. Neutron diffraction investigation upon heating at ambient pressure}

In order to probe the crystal structure changes occurring above room temperature, the $\mathrm{YFe}_{2}$ compound has been heated and neutron diffraction patterns recorded in situ through the furnace. Figure 2 illustrates the different powder neutron 


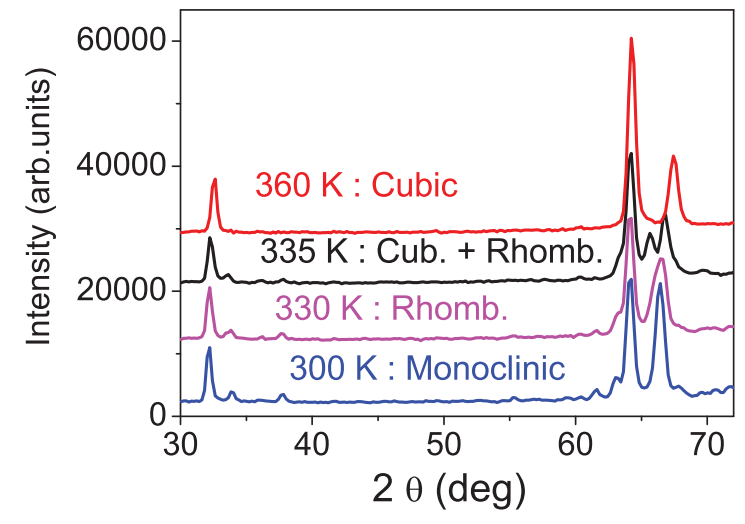

FIG. 2. (Color online) Neutron diffraction patterns recorded at ambient pressure and the indicated temperatures for $\mathrm{YFe}_{2} \mathrm{D}_{4.2}$. The corresponding crystal structure lattices are also indicated.

diffraction patterns recorded in each temperature range. In agreement with X-ray diffraction (XRD) data reported in Ref. 8, we observed two successive structural transitions. First, the monoclinic structure, observed at room temperature and below, transforms into the rhombohedral one at about $323 \mathrm{~K}$. As can be seen from the Fig. 2, this manifests itself by the disappearance of small diffraction peaks, in particular the $(-312)$ located slightly above $62^{\circ}(2 \theta)$. Then the rhombohedral $R-3 m$ symmetry is preserved up to about $343 \mathrm{~K}$ where the crystal structure becomes more symmetric, exhibiting a cubic lattice. Some structural parameters derived from the Rietveld refinement of the neutron diffraction pattern plotted in Fig. 2 are summarized in Table I together with the agreement factors of the refinement as well as the percentage of each phase. More detailed information as well as the Rietveld refinement plots are given in the Supplementary Material ${ }^{26}$ (Fig. S1). Structural details used for the Rietveld analysis are also listed in Tables S1 to S3 of the Supplementary Material. ${ }^{26}$

The analysis of the powder diffraction patterns demonstrates the existence of a significant temperature range of coexistence of the rhombohedral and the cubic lattices. This is well illustrated by the evolution of the percentages of each phase plotted in Fig. 3. The thermal evolutions of the $a$ and $c$ lattice parameters of the rhombohedral structure are given in Fig. 4. It is remarkable that these parameters are first slowly evolving, but then exhibit a much sharper evolution close to the transition into cubic structure. It is worth recalling that the origin of these structural transitions is due to ordering of deuterium near room temperature within the metallic crystal structure.
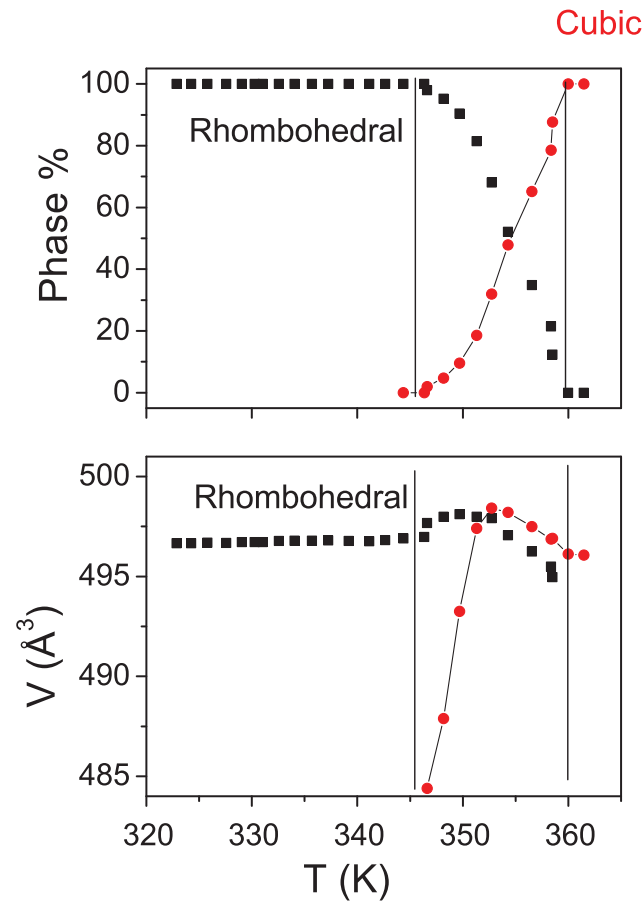

FIG. 3. (Color online) Thermal evolution of the phase content along the structural transition from rhombohedral (square) to cubic (circle) transformation of $\mathrm{YFe}_{2} \mathrm{D}_{4.2}$ derived from neutron diffraction pattern refinement.

\section{B. X-ray diffraction investigation under applied pressure}

In order to probe the pressure dependence of the crystal structure, the energy dispersive $\mathrm{x}$-ray diffraction patterns have been recorded for several applied pressures ranging from 0 to $5 \mathrm{GPa}$. The corresponding diffraction patterns are compared in Fig. 5(a). For comparison, Fig. 5(b) presents a simulation of the x-ray diffraction patterns for the three different types of crystal structure. A broad line width has been used to match the experimental pattern. It is worth noticing that due to the limited resolution of these diffraction experiments, the monoclinic distortion of the $\mathrm{YFe}_{2} \mathrm{D}_{4.2}$ could not be resolved. Consequently, the x-ray diffraction patterns have been analyzed in the rhombohedral approximant related cell. The relation between the monoclinic volume and the rhombohedral volume (in hexagonal setting) is as follows: $V_{\text {mono }}(C 2 / m)=4 / 3 V_{\text {hexa }}(R-3 m)$. A relationship between the cubic, rhombohedral, and monoclinic cells are given in Table II (see Ref. 8 for more details). The corresponding crystal structures are compared in Fig. 1. The rhombohedral structure

TABLE I. Structural parameters as derived from the Rietveld refinement of the neutron powder diffraction patterns plotted on Fig. 2 and recorded at the indicated temperatures.

\begin{tabular}{|c|c|c|c|c|c|c|c|c|c|}
\hline$T(\mathrm{~K})$ & Struct. & $a(\AA)$ & $b(\AA)$ & $c(\AA)$ & $\beta\left({ }^{\circ}\right)$ & $V\left(\AA^{3}\right)$ & $\mathrm{Wt}_{\%}$ & $\mathrm{R}_{\text {Bragg\% }}$ & $\mathrm{R}_{\mathrm{wp} \%}$ \\
\hline 300 & Mono. & $5.487(1)$ & $11.4264(23)$ & $9.3960(14)$ & $122.48(11)$ & $496.98(16)$ & 100 & 11.6 & 14.3 \\
\hline 330 & Rhomb. & $5.7053(7)$ & & $13.2407(18)$ & & $373.24(82)$ & 100 & 4.06 & 15.0 \\
\hline \multirow[t]{2}{*}{335} & Rhomb & $5.688(1)$ & & $13.3307(2)$ & & $373.483(96)$ & 81.5 & 10.1 & 14.9 \\
\hline & Cubic & $7.923(2)$ & & & & $497.37(24)$ & 18.5 & 15.7 & \\
\hline 360 & Cubic & $7.9161(6)$ & & & & $496.061(70)$ & 100 & 6.85 & 12.6 \\
\hline
\end{tabular}



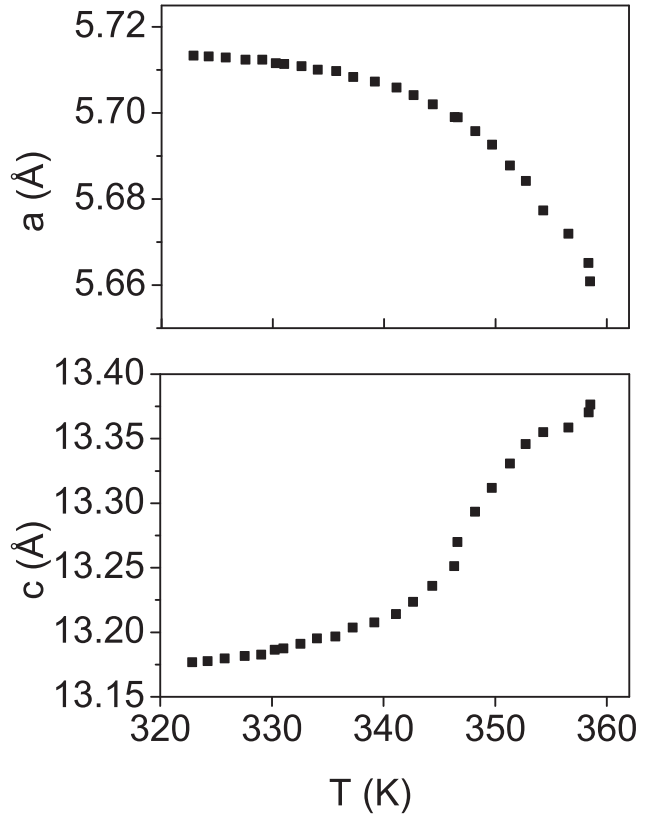

FIG. 4. Thermal evolution of the lattice parameters of $\mathrm{YFe}_{2} \mathrm{D}_{4.2}$ in the temperature range where the rhombohedral structure is retained. The results are derived from neutron powder diffraction analysis.

is described in the corresponding hexagonal triple cell, which is related to the cubic one by the following relations:

$$
\begin{aligned}
& a_{\text {hexa }} \approx \sqrt{2} a_{c}, \\
& c_{\text {hexa }} \approx \sqrt{3} a_{c},
\end{aligned}
$$

where the index hexa and $c$ refer to the hexagonal and cubic cell, respectively.

The XRD patterns under pressure [Fig. 5(a)] show that the ambient pressure crystal structure of $\mathrm{YFe}_{2} \mathrm{D}_{4.2}$ is maintained up to $4 \mathrm{GPa}$ with a progressive decrease of the cell parameters. This decrease is linear, and values of $\Delta a / a=1.91 \%$ and $\Delta c / c=1.46 \%$ have been obtained from the fit from 0 to 4 GPa of the data given in Fig. 6. This indicates an anisotropic change of the lattice with a larger compression in the basal plane. The cell volume decrease in the monoclinic range at $4.76 \AA^{3} \mathrm{GPa}^{-1}$, corresponding to a relative volume change of $-1.26 \%$.

These values correspond to a cell volume decrease up to $4 \mathrm{GPa}$ of $5.2 \%$ with a compressibility value of $\kappa=$ $0.013 \mathrm{GPa}^{-1}$. This value is slightly larger but well compa-

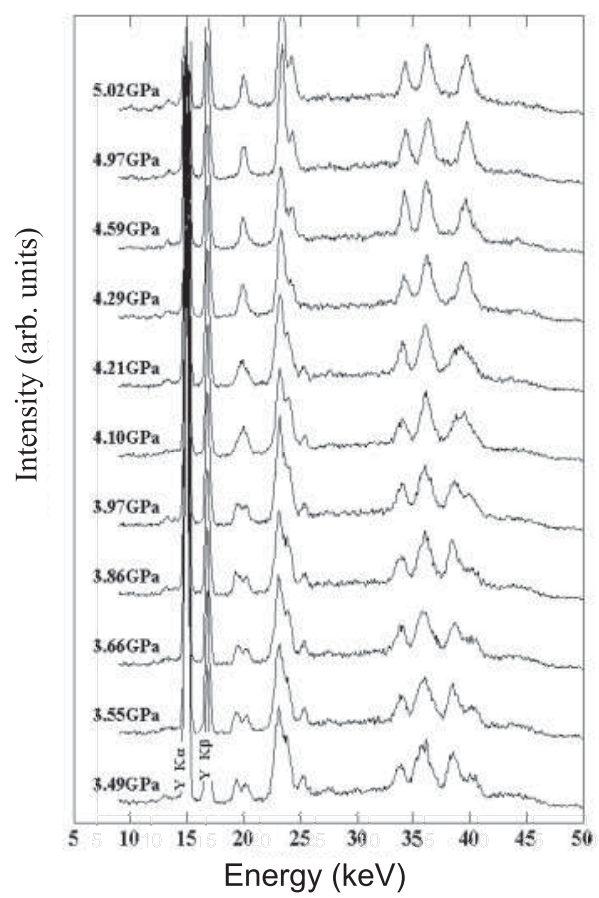

(a)

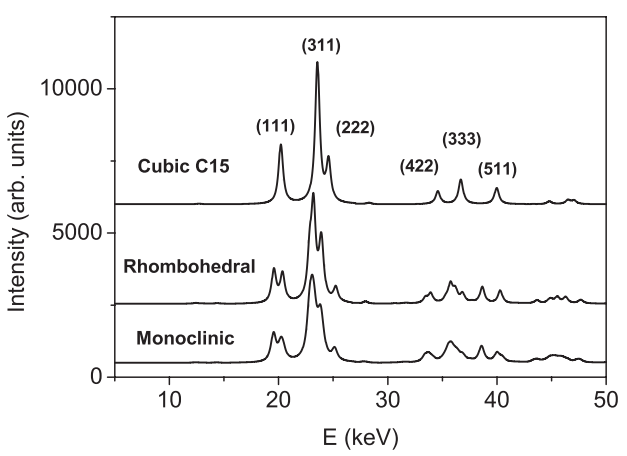

(b)

FIG. 5. Above (a): x-ray diffraction patterns recorded at room temperature for $\mathrm{YFe}_{2} \mathrm{D}_{4.2}$ at different applied pressures between 3.5 and $5 \mathrm{GPa}$. The cubic structure is observed for pressures starting from 4.29 $\mathrm{GPa}$ and above. Below (b): simulation of XRD patterns for the three type of structures.

rable with compressibility value of $\mathrm{YFe}_{2}$ compound $(\kappa=$ $\left.0.011 \mathrm{GPa}^{-1}\right)$ and $\mathrm{YFe}_{2} \mathrm{H}_{5}\left(\kappa=0.010 \mathrm{GPa}^{-1}\right) .{ }^{27}$ It is worth mentioning that up to $5 \mathrm{GPa}$, other $\mathrm{ErFe}_{2} \mathrm{D}_{x}$ compounds with

TABLE II. Cell description of $\mathrm{YFe}_{2} \mathrm{D}_{4.2}$ in the various crystal structures that this compound adopts as a function of temperature and pressure. The vectors for the rhombohedral and monoclinic structures are expressed as a function of the cubic cell. The volume is expressed as a function of the various cell parameters which can be refined for each structure.

\begin{tabular}{lcccc}
\hline \hline Parameter & Cubic & Rhombohedral (hexagonal) & Monoclinic nuclear cell & Monoclinic magnetic cell AFM (2b) \\
\hline Space group & $F d-3 m$ & $R-3 m$ & $P c$ & $P c$ \\
$\vec{a}$ & $\vec{a}_{c}$ & $\approx \frac{1}{2}\left(\vec{a}_{c}-\vec{c}_{c}\right)$ & $\approx \frac{1}{2}\left(\vec{a}_{c}+\vec{b}_{c}\right)$ & $\approx \frac{1}{2}\left(\vec{a}_{c}+\vec{b}_{c}\right)$ \\
$\vec{b}$ & $\vec{b}_{c}$ & $\approx \frac{1}{2}\left(-\vec{a}_{c}+\vec{b}_{c}\right)$ & $\approx 2\left(\vec{a}_{c}-\vec{b}_{c}\right)$ \\
$\vec{c}$ & $\vec{c}_{c}$ & $\approx \vec{a}_{c}+\vec{b}_{c}+\vec{c}_{c}$ & $\approx-\frac{1}{2}\left(\vec{a}_{c}+\vec{b}_{c}+2 \vec{c}_{c}\right)$ & $\approx-\frac{1}{2}\left(\vec{a}_{c}+\vec{b}_{c}+2 \vec{c}_{c}\right)$ \\
$V$ & $a_{c}^{3}$ & $\frac{\sqrt{3}}{2} a_{R}^{2} \cdot c_{R}$ & $a_{M} \cdot b_{M} \cdot c_{M} \cdot \sin \beta$ & $2 \cdot a_{M} \cdot b_{M} \cdot c_{M} \cdot \sin \beta$ \\
$Z$ & 8 & 6 & 8 & 16 \\
\hline \hline
\end{tabular}




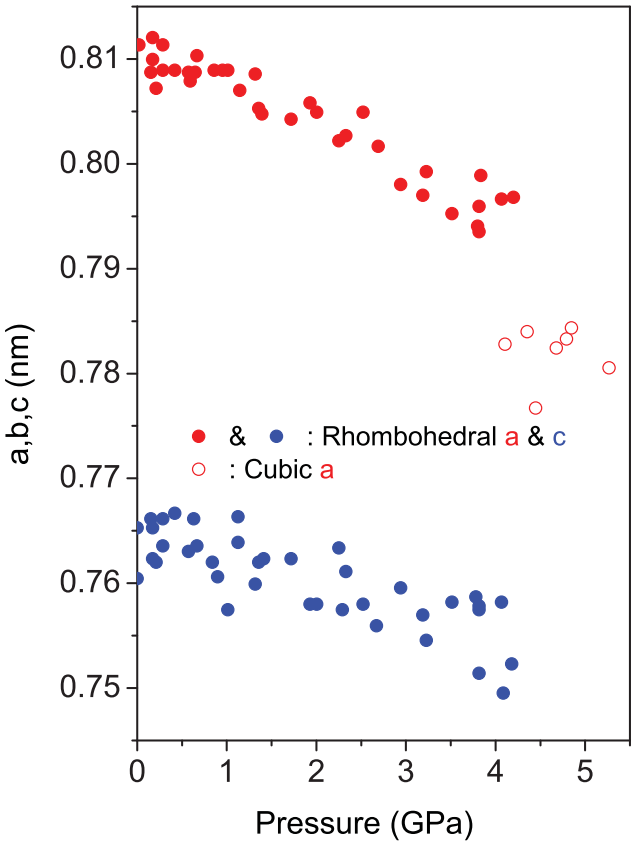

FIG. 6. (Color online) Cell parameter variation of $\mathrm{YFe}_{2} \mathrm{D}_{4.2}$ versus applied pressure.

intermediate interstitial contents also exhibit a linear pressure dependence of the unit cell volume ${ }^{28}$ with a similar slope, indicating that the compressibility of these compounds is similar. At 4.2 $\mathrm{GPa} \mathrm{YFe}_{2} \mathrm{D}_{4.2}$ undergoes a transition from a monoclinic to a cubic structure (Fig. 6). Such remarkable change of crystal symmetry is not surprising if one recalls:

(i) that the original $\mathrm{YFe}_{2}$ intermetallic compound is of cubic $\mathrm{C} 15$ symmetry, and

(ii) that the $\mathrm{YFe}_{2} \mathrm{D}_{x}$ compounds undergo a transition toward the cubic $\mathrm{C} 15$ structure above a transition temperature which depends upon the $\mathrm{D}$ content $(1.2<x \leqslant 4.2) .^{5,8}, 11$

For comparison purposes, some unit cell parameters of the $\mathrm{YFe}_{2} \mathrm{D}_{4.2}$ compound derived from fit at the different temperature and pressures are listed in Table III. At room temperature and $4 \mathrm{GPa}$, the phase transition observed could be interpreted as reminiscent of the hydrogen order-disorder transition known to occur at $345 \mathrm{~K}$ at ambient pressure. In this case, the effect of an applied pressure can be the lowering of the order-disorder transition temperature below room temperature. ${ }^{4}$ Note that this behavior is different from that of $\mathrm{YMn}_{2} \mathrm{D}_{4.3}$, where an increase of the rhombohedral distortion is observed upon pressure. ${ }^{2}$

\section{Neutron diffraction study under pressure}

At ambient pressure, the neutron powder diffraction pattern of $\mathrm{YFe}_{2} \mathrm{D}_{4.2}$ displays a ferromagnetic structure below $T_{M 0}$ and an AFM structure between $T_{M 0}$ and $T_{N}$. The low-angle part of the neutron diffraction patterns recorded at ambient pressure are compared in Fig. 7(a) for 1.5, 90, and $180 \mathrm{~K}$. The AFM structure can be described by doubling the nuclear cell along the $b$ axis and is characterized by an intense peak at $d=$ $23.5 \AA$ and a smaller one at $d=5.51 \AA$. In this new cell $\left(b^{\prime}=2 b\right)$, the AFM peaks are indexed as $(010)$ and $(031)$, respectively, whereas the main FM peak is indexed as (100). Due to the large wavelength $(\lambda=4.7 \AA)$ and also the weak signal-to-noise ratio, the neutron patterns measured on G61 display mainly the (010) AFM line at $2 \theta=10.8^{\circ}(d=23.5 \AA)$ and the (100) and (002) nuclear lines at $2 \theta=60^{\circ}(d=4.64 \AA)$ and at $2 \theta=72^{\circ}(d=3.97 \AA)$, respectively [Fig. 7(b)]. An additional FM contribution can be observed on the (100) peak as seen on the difference curve. The contribution at $2 \theta=19^{\circ}$ is not a Bragg peak but a background contribution. The evolution of the FM and AFM magnetic contributions in Figs. 8 and 9 was determined by subtracting the pattern at $180 \mathrm{~K}$, which is above the Néel temperature, and normalizing the patterns to the nuclear contribution. The values at $0 \mathrm{GPa}$ were those measured previously on the G41 spectrometer of the LLB. At $0 \mathrm{GPa}$, the FM-AFM transition temperature $T_{M 0}$ is $84 \mathrm{~K}$ and the Néel temperature $T_{N}$ is at $131 \mathrm{~K}$. At $0.5 \mathrm{GPa}$, the FM component decreases with $T_{M 0}$ close to $36 \mathrm{~K}$. The AFM line is observed at $1.5 \mathrm{~K}$, decreases slightly with a minimum at $50 \mathrm{~K}$, increases again with a maximum at $70 \mathrm{~K}$ and disappears above $T_{N}=125 \mathrm{~K}$. This behavior can be explained considering a mixture of two phases, one where the AFM is formed at low temperature and a second one with $T_{M 0}=36 \mathrm{~K}$ and $T_{N}=$ $125 \mathrm{~K}$. For $P=1,2.6$, and $4 \mathrm{GPa}$, there is no ferromagnetic contribution, and the AFM Bragg peak decreases upon increasing temperature from $125 \mathrm{~K}$ to $T_{N}=90 \mathrm{~K}$. $T_{N}$ is not very sensitive to the pressure above $1 \mathrm{GPa}$. The AFM line intensity versus temperature can be fitted according to:

$$
I=I_{0}+A^{*}\left(T-T_{M 0}\right)^{\tau} .
$$

For all the curves, the exponent $\tau$ is very close to $3 / 2$ and $A$ is negative, which means that the behavior is close to a Bloch function. In a previous study, ${ }^{12}$ it was found that the

TABLE III. Refined unit cell parameters of the $\mathrm{YFe}_{2} \mathrm{D}_{4.2}$ compound measured at the indicated temperatures and pressures.

\begin{tabular}{|c|c|c|c|c|c|}
\hline Parameter & Cubic & Rhombohedral (hexagonal) & Monoclinic nuclear cell & & \\
\hline Space group & $F d-3 m$ & $R-3 m$ & $P c$ & & \\
\hline Pressure (GPa) & Ambient pressure & $P=4 \mathrm{GPa}$ & Ambient pressure & $P=3 \mathrm{GPa}$ & Ambient pressure \\
\hline Temperature (K) & 360 & 300 & 330 & 300 & 290 \\
\hline$a(\AA)$ & 7.95 & 7.85 & 5.710 & 5.657 & 5.5066 \\
\hline$b(\AA)$ & 7.95 & 7.85 & 5.710 & 5.657 & 11.482 \\
\hline$c(\AA)$ & 7.95 & 7.85 & 13.18 & 13.164 & 9.4292 \\
\hline$\beta\left({ }^{\circ}\right)$ & & & & & 122.3 \\
\hline$V\left(\AA^{3}\right)$ & 502.5 & 483.7 & 372.2 & 364.8 & 503.76 \\
\hline Equivalent $V\left(\AA^{3}\right)$ in monoclinic & 502.5 & 483.7 & 496.8 & 486.4 & 503.8 \\
\hline
\end{tabular}




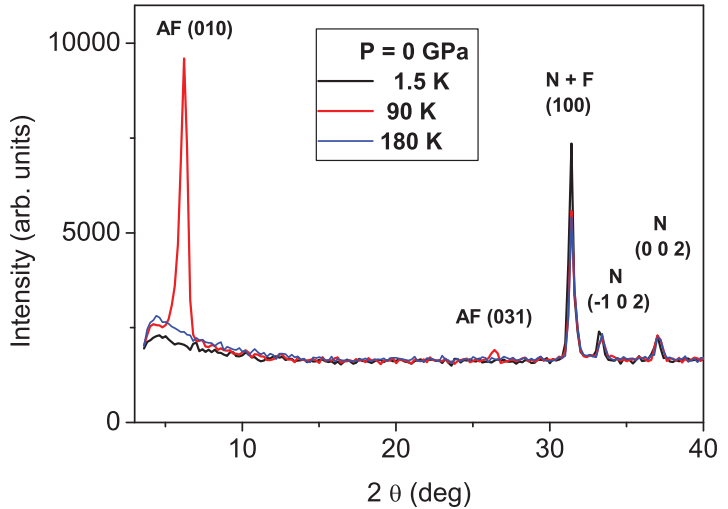

(a)

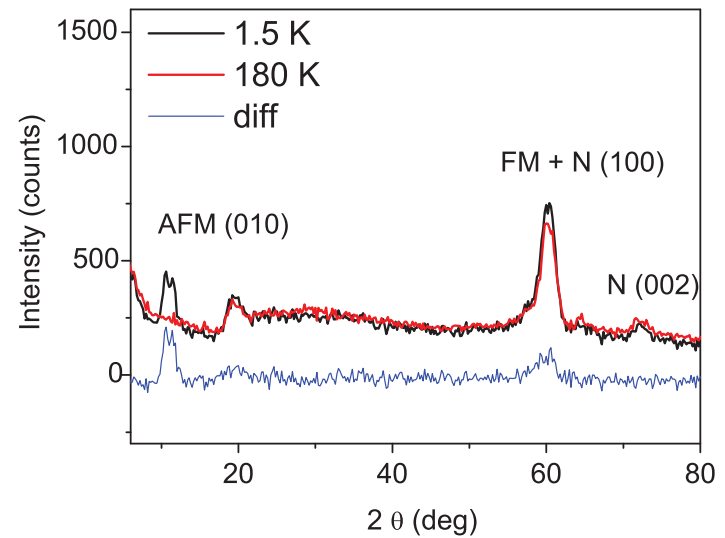

(b)

FIG. 7. (Color online) (a) Neutron diffraction patterns of $\mathrm{YFe}_{2} \mathrm{D}_{4.2}$ measured at ambient pressure on $\mathrm{D} 1 \mathrm{~B}$. The $2 \theta$ range presented here corresponds to that measured on G6.1 taking into account the different wavelengths (2.523 and $4.70 \AA$, respectively). Fig. 7(b) Neutron patterns of $\mathrm{YFe}_{2} \mathrm{D}_{4.2}$ under $0.5 \mathrm{GPa}$ and at 1.5 and $180 \mathrm{~K}$ and corresponding difference curve.

AFM structure is constituted by FM layers of Fe with moments parallel to the $(a, c)$ plane and with an inversion of the direction of the Fe moment below and above the layer which contains the Fe atom which looses its moment at $T_{M 0}$ through an IEM behavior. $^{12}$ The variation of the AFM intensity can be an indication that the Fe moments belonging to these intermediate FM layers are decreasing according to the Bloch function.

In Fig. 10, are reported the evolution of $T_{M 0}$ and $T_{N}$ as a function of the applied pressure. In this figure, we observe a continuous and progressive decrease of $T_{M 0}$ with the pressure. This transition has already disappeared at $1 \mathrm{GPa}$ since the ferromagnetic contribution to the Bragg peak has vanished. $T_{N}$ decreases also up to $1 \mathrm{GPa}$ and is not very sensitive to the pressure above. At a given temperature, the intensity of the ferromagnetic Bragg reflections decreases upon increasing the applied pressure. Even if $T_{N}$ remains the same, this is a clear indication that the Fe magnetic moments are decreasing, whereas the AFM coupling is preserved up to pressure as high as $4 \mathrm{GPa}$, that is to say in the whole monoclinic domain. It is worth noticing that $T_{N}$ and $T_{M 0}$ both decrease under pressure, and $T_{N}$ saturates when $T_{M 0}$ goes to zero. At low temperature, the decrease of $T_{N}$ and $T_{M 0}$ are clearly correlated, indicating that the appearance of ferromagnetic order at low temperature

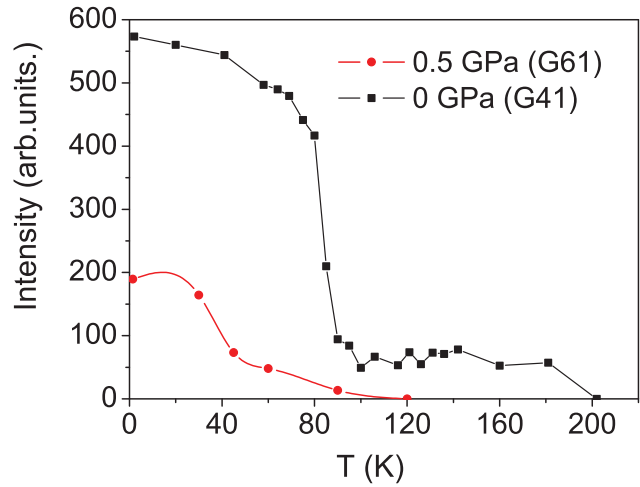

FIG. 8. (Color online) Thermal variation of the ferromagnetic (001) line intensity of $\mathrm{YFe}_{2} \mathrm{D}_{4.2}$ at 0 and $0.5 \mathrm{GPa}$ measured by neutron powder diffraction on the diffractometer G4.1 and G6.1.

$(T<100 \mathrm{~K})$ and pressure $(P<1 \mathrm{GPa})$ does favor larger ordering temperature, even in the AFM state. For $P \geqslant 1 \mathrm{GPa}$, where only the AFM structure remains, $T_{N}$ is not sensitive to the applied pressure. The FM-AFM transition at $T_{M 0}$ is clearly sensitive to the volume change induced by the applied pressure due to its IEM character.

This behavior is similar to that of $\mathrm{Hf}_{0.9} \mathrm{Ta}_{0.1} \mathrm{Fe}_{2}$ under pressure ${ }^{14}$ which has been reported to present a first-order phase transition from ferromagnetic to antiferromagnetic states above $0.86 \mathrm{GPa}$. Nishihara has also reported similar phase transition in related $\mathrm{AFe}_{2}$ systems ${ }^{29}$ and pointed out the importance of the spin fluctuation in such systems.

At this point, it is worth recalling that in several other densely packed iron compounds, drastic change of the magnetic state has been observed around a critical value of $\mathrm{Fe}-\mathrm{Fe}$ distance. ${ }^{30-33}$ A slight increase of such distance in a narrow range around the critical value $(\sim 2.5 \AA)$ leads to change from AFM to FM ground state with respectively low volume and low spin moment and high volume and high spin moment. Such change of the magnetic state has, for example, been induced by pressure in the $\mathrm{La}\left(\mathrm{Fe}_{0.8} \mathrm{Al}_{0.12}\right)_{13}$ compound, ${ }^{33}$ triggering an instability of the Fe magnetic moment. Fujita et al. ${ }^{34}$ reported recently that the critical pressure for the disappearance of the FM state is about $1.3 \mathrm{GPa}$ in the $\mathrm{La}\left(\mathrm{Fe}_{0.89} \mathrm{Si}_{0.11}\right)_{13}$ compound, a critical pressure value close to the $1 \mathrm{GPa}$ found here for $\mathrm{YFe}_{2} \mathrm{D}_{4.2}$.

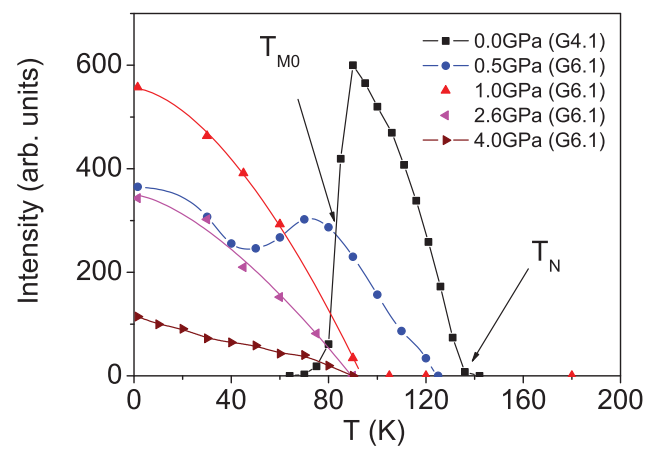

FIG. 9. (Color online) Thermal evolution of the (010) AFM line for different applied pressures measured by neutron powder diffraction as measured on the instruments G6.1 and G4.1. 


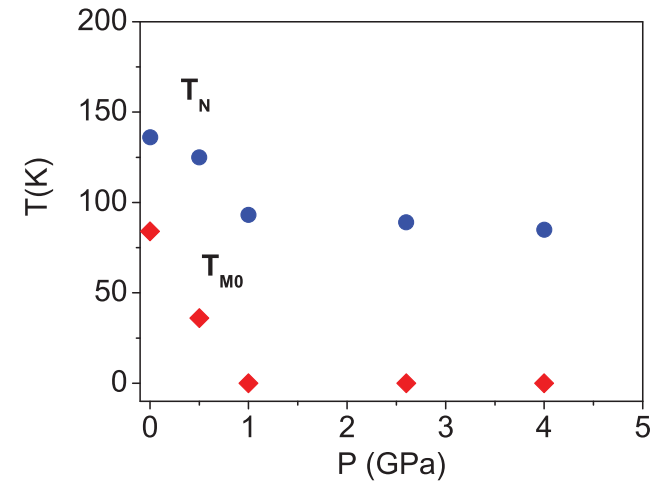

FIG. 10. (Color online) Evolution of the transition temperatures ( $T_{N}$ and $T_{M 0}$ ) versus pressure estimated from high-pressure neutron measurements.

\section{MAGNETIC MEASUREMENTS UNDER PRESSURE}

Temperature dependencies of the low-field magnetization measured at a magnetic field of $0.03 \mathrm{~T}$ under different pressure values are displayed in Fig. 11. It is easy to see that the magnetic ordering temperature $T_{M 0}$ dramatically decreases with increasing pressure and with a slope $d T_{M 0} / d P=$ $-156 \mathrm{~K} \mathrm{GPa}^{-1}$, as is illustrated in Fig. 12. The magnetic ordering temperature is completely suppressed by a pressure of about $0.54 \mathrm{GPa}$. Using the compressibility value determined in this work, $\kappa=0.013 \mathrm{GPa}^{-1}$, it can be determined that a relative volume decrease larger than $0.70 \%(0.23 \%$ of lattice parameter) changes the ground state of $\mathrm{YFe}_{2} \mathrm{D}_{4.2}$ deuteride from FM to AFM state.

Figure 13 presents a set of magnetization isotherms as a function of magnetic field at atmospheric pressure. These results are in agreement with those published in Refs. 12 and 21. The critical field $\left(H_{C}\right)$ rapidly increases with temperature with slope $d H_{C} / d T=0.17 \mathrm{~T} \mathrm{~K}^{-1}$, reaching our maximum attainable field of $7 \mathrm{~T}$ around $125 \mathrm{~K}$, about $40 \mathrm{~K}$ above the low field transition temperature. Note that this value is slightly larger than the slope $d H_{C} / d T=0.14 \mathrm{~T} \mathrm{~K}^{-1}$ reported in Ref. 12. A detailed analysis of this phenomenon will be presented elsewhere.

Sets of magnetization isotherms as a function of magnetic field at several pressures are displayed in Figs. 14(a), 14(b) and 14(c), respectively. Due to the large pressure effect on $T_{M 0}$ and remarkable changes of the shape of magnetic isotherms, the

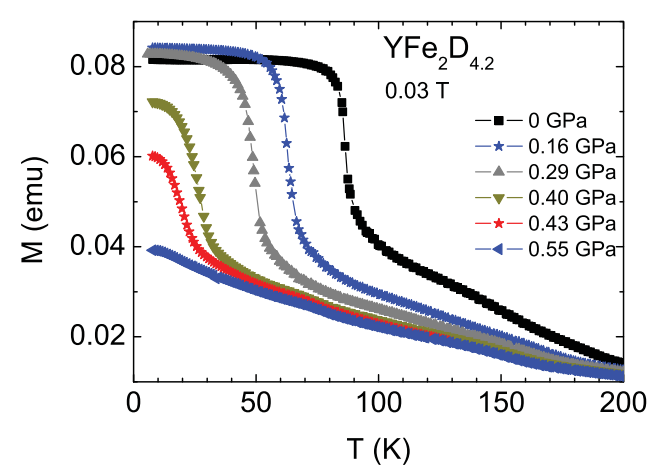

FIG. 11. (Color online) Thermal evolution of the magnetization recorded for several applied pressures on $\mathrm{YFe}_{2} \mathrm{D}_{4.2}$.

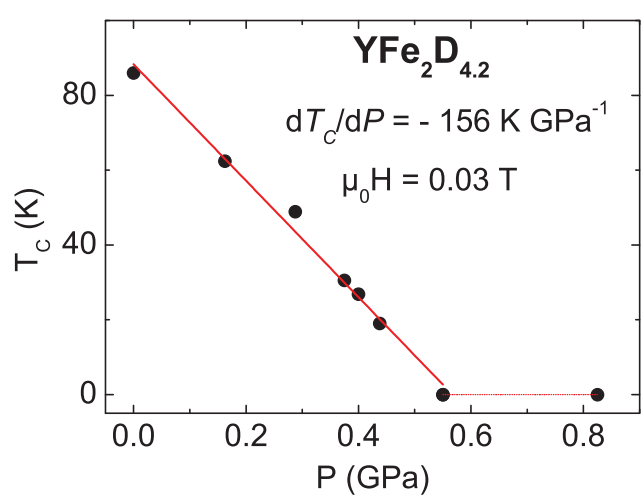

FIG. 12. (Color online) Pressure dependence of the Curie temperature as derived from isofield magnetization curves of $\mathrm{YFe}_{2} \mathrm{D}_{4.2}$.

determination of the pressure effect on spontaneous magnetization $M_{S}$ can be determined only at pressures below $0.4 \mathrm{GPa}$. The pressure induced decrease of $M_{S}, d \ln \left(M_{S}\right) / d P=-7.3$ $\times 10^{-2} \mathrm{GPa}^{-1}$ is remarkably higher than the value for pure $\alpha$-Fe $\left(-4.2 \times 10^{-3} \mathrm{GPa}^{-1}\right.$ experimental value, close to the theoretical value of $-5 \times 10^{-3} \mathrm{GPa}^{-1}$ ) but compares well with the values of other binary Fe-based intermetallic compounds $\left(d \ln \left(M_{S}\right) / d P=-8.2 \times 10^{-2} \mathrm{GPa}^{-1}\right.$ for $\left.\mathrm{Y}_{2} \mathrm{Fe}_{17}\right){ }^{35-37}$ The pressure induced decrease of $M_{S}$ observed here for $\mathrm{YFe}_{2} \mathrm{D}_{4.2}$ is about one order of magnitude higher than what has been reported for the starting $\mathrm{YFe}_{2}$ compound by Armitage et al.:.$^{38}$ $d \ln \left(M_{S}\right) / d P=-8.4 \times 10^{-3} \mathrm{GPa}^{-1}$.

The critical field $\left(H_{C}\right)$ at a given temperature rapidly increases with increasing pressure, as expected due to the large negative pressure-induced shift of the transition temperature.

Magnetic measurements indicates that $T_{M 0}$ reaches zero for a pressure of $0.54 \mathrm{GPa}$, whereas the neutron diffraction shows that part of the sample is still a ferromagnetic with $T_{M 0}=36 \mathrm{~K}$ at $0.5 \mathrm{GPa}$. This discrepancy can be related to the fact that the pressure is better calibrated in the range studied by magnetic measurements. In addition, the neutron pattern evolution showed a mixture of two phases: one which already has an AFM ground state, whereas the second one undergoes the FM-AFM transition at $36 \mathrm{~K}$. It can, however, reveal a first-order behavior (coexistence of two phases with a large hysteresis effect) or an inhomogeneity in the applied pressure inside the sample. But even if the precision on the

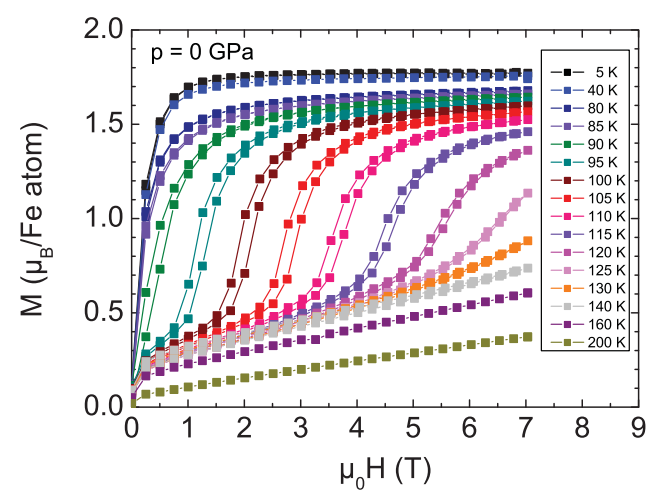

FIG. 13. (Color online) Isothermal magnetization curves recorded at the indicated temperature in the high-pressure cell, result in agreement with Ref. 21. 


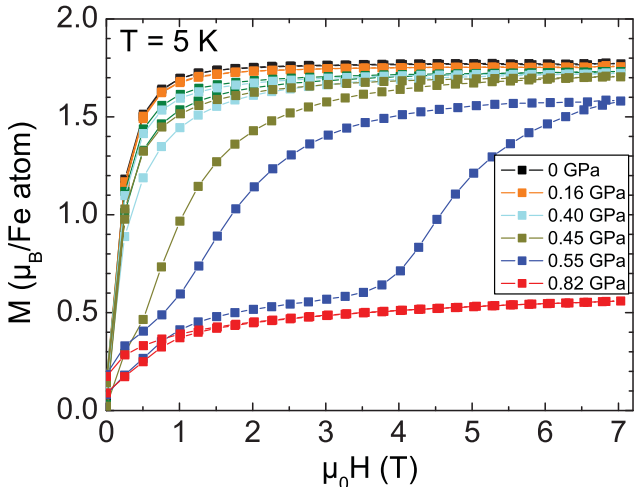

(a)

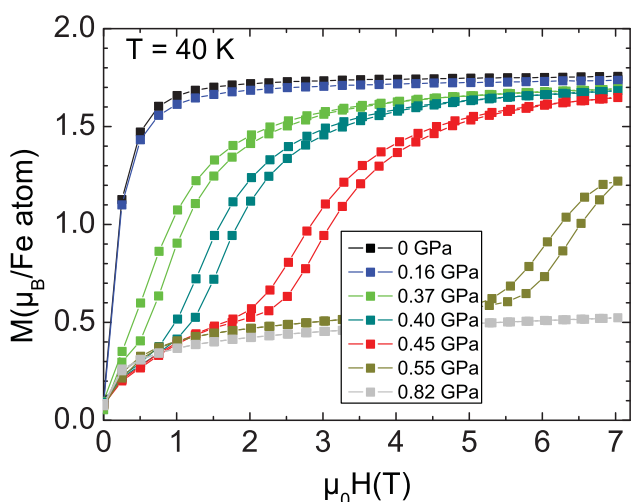

(b)

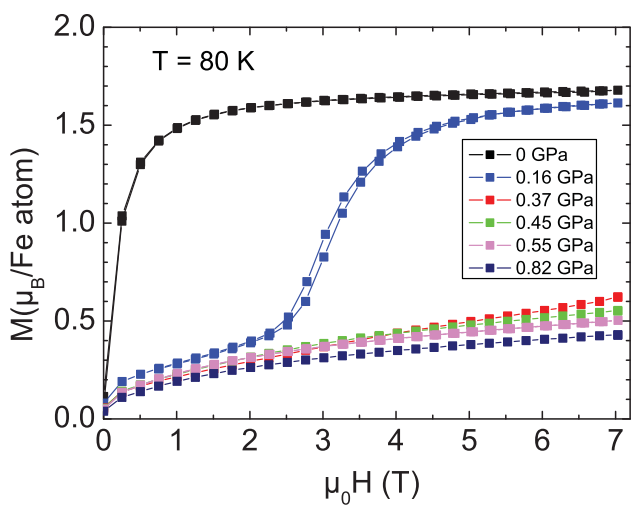

(c)

FIG. 14. (Color online) Isothermal magnetization curves recorded at (a) 5, (b) 40, and (c) $80 \mathrm{~K}$ for the indicated applied pressures.

pressure calibration is smaller with neutrons than with present magnetic measurements, it has the advantage to extend to higher pressure (4 GPa) and to bring information on the AFM phase, in particular the determination of $T_{N}$. Thanks to the high-pressure XRD, we can underline that no structural change has been observed to occur when the ferromagnetic order is suppressed.

Another iron-lanthanide intermetallic hydride $\mathrm{La}(\mathrm{Fe}, \mathrm{Si})_{13} \mathrm{H}_{x}$ has been recently found to exhibit a peculiar series of consecutive field-induced transitions. The pressure-induced discontinuous magnetization jumps have resulted in an enhanced cooling power leading to exceptional magnetocaloric properties around room temperature for these hydrides. ${ }^{39,40}$ Unlike the starting $\mathrm{LaFe}_{11.6} \mathrm{Si}_{1.4}$ compounds, which also present an itinerant electron metamagnetic transition, the IEM transition at zero applied pressure being even sharper than for the parent hydrides, $\mathrm{YFe}_{2}$ is known to exhibit a classic ferromagnetic behavior up to its ordering temperature. The strong reduction of the Curie temperature from $540 \mathrm{~K}$ down to about $84 \mathrm{~K}$ (for $\mathrm{YFe}_{2}$ and $\mathrm{YFe}_{2} \mathrm{D}_{4.2}$, respectively) upon deuterium absorption bears witness to the evolution of the Fe magnetism towards more delocalized character in the sense of Rhodes and Wolfarth plots. ${ }^{41}$ This is in excellent agreement with the observed hydrogen-induced metamagnetic transition typical of an itinerant electron behavior. ${ }^{42,43}$ The fact that the ferromagnetic order disappears upon applying pressure on $\mathrm{YFe}_{2} \mathrm{D}_{4.2}$, whereas the opposite has been reported for the $\mathrm{YFe}_{2}$ compound with $d T_{C} / d P=5 \mathrm{~K} \mathrm{GPa}^{-1}$ (Ref. 44) is a further proof of the large evolution of the magnetic character of the iron sublattice upon deuterium insertion.

Previous band structure calculations have shown that, in $\mathrm{YFe}_{2}$ hydrides, there is a large competition between the volume effect and the chemical effect related to $\mathrm{Fe}-\mathrm{H}$ bondings. ${ }^{10}$ Increasing the volume leads to a better localization of the $\mathrm{Fe}$ $3 d$ bands and therefore an increase of the Fe moment. On the contrary, the $\mathrm{H}$ insertion weakens the Fe-Fe interactions $\left(T_{C}\right.$ decrease) and the Fe moment for $x>3.5$. In this paper, it is clearly observed that for a given $D(H)$ content, decreasing the volume by applying an external pressure lowers both the Fe-Fe interaction ( $T_{M 0}$ decrease) and the Fe moment. This effect is most probably enhanced by the IEM behavior observed for $\mathrm{YFe}_{2} \mathrm{D}_{4.2}$.

\section{CONCLUSIONS}

We have established the pressure evolution of the $\mathrm{YFe}_{2} \mathrm{D}_{4.2}$ crystal structure up to $5.5 \mathrm{GPa}$, showing a structural phase transition at $4 \mathrm{GPa}$ from the monoclinic symmetry (retained at ambient pressure) to the cubic one. The magnetic phase diagram has been determined from 2 to $300 \mathrm{~K}$ and 0 to $4 \mathrm{GPa}$ by a combination of a high-pressure neutron diffraction experiment and high-pressure magnetization measurement. At ambient pressure, a ferromagnetic order is observed at low temperature as a ground state, upon increasing it gives rise to antiferromagnetic ordering above $T_{M 0}=84 \mathrm{~K}$ an order that disappears at $T_{N}=136 \mathrm{~K}$. Both $T_{N}$ and $T_{M 0}$ are decreasing upon applying an external pressure. The ferromagnetic order disappears at a pressure of $0.54 \mathrm{GPa}$, the pressure induced decrease of the ordering temperature is huge, $\partial T_{M 0} / \partial P=$ $-156 \mathrm{~K} \mathrm{GPa}^{-1}$. The crucial role of the volume effect was demonstrated by the close agreement of the decrease of volume at the critical pressure $(-0.70 \%$ at $0.54 \mathrm{GPa})$ with the increase of volume observed at the transition from antiferromagnetic to ferromagnetic state at ambient pressure $-0.65 \%$. We consequently can conclude that pressure destabilizes the ferromagnetic order in $\mathrm{YFe}_{2} \mathrm{D}_{4.2}$ a behavior similar to what has been reported in $\mathrm{R}_{2} \mathrm{Fe}_{17}$ or $\mathrm{R}_{2} \mathrm{Fe}_{17} \mathrm{H}_{x}$ compounds. ${ }^{35,37}$ This paper has demonstrated the large coupling between magnetic and structural properties of $\mathrm{YFe}_{2} \mathrm{D}_{4.2}$ and in particular the high pressure sensitivity of the physical properties. The Fe magnetic behavior of the $\mathrm{Fe}$ sublattice is clearly of itinerant electron type here and exhibits a large delocalized character which contrasts the well-known localized behavior of pure iron or the rather localized behavior of the starting $\mathrm{YFe}_{2}$ intermetallic 
compound. The itinerancy observed here is closer to the behavior previously reported for some other $\mathrm{AFe}_{2}$ compounds $A=\mathrm{Ta}, \mathrm{Hf}^{13-16,29}$ as well as that of other Fe rich compounds such as the $\mathrm{La}\left(\mathrm{Fe}_{0.89} \mathrm{Si}_{0.11}\right)_{13}$ compound. ${ }^{34}$

As discussed above, the delocalized character of $\mathrm{Fe}$ in the hydride contrasts with the localized character in $\mathrm{YFe}_{2}$. This result differs from the behavior in the $\mathrm{YMn}_{2}-\mathrm{YMn}_{2} \mathrm{D}_{4}$. A comparison between the two systems has been published previously ${ }^{45}$ on the basis of experimental results. The hydrogen insertion in $\mathrm{YMn}_{2}$ increases the cell volume and stabilizes the Mn moment, which becomes more localized due to the increase of $\mathrm{Mn}-\mathrm{Mn}$ distances. The bonding between $\mathrm{Mn}$ and $\mathrm{H}$ atoms tend to shift the Fermi level to larger values, i.e. in a range where the density of states (DOS) will increase. ${ }^{46}$ Therefore, both volume and electronic effect tend to stabilize the Mn moment. In $\mathrm{YFe}_{2}$, the ferromagnetic state is stable, and the DOS at $E_{F}$ is elevated. Upon $\mathrm{H}$ absorption in this compound, there is a competition between two effects. The increase of cell volume stabilizes the Fe moment, but the $\mathrm{Fe}-\mathrm{H}$ bonding has a destabilization effect. ${ }^{10}$ For $5 \mathrm{H} /$ f.u., the energy of the nonpolarized state is lower than the spin polarized state. For $x=4.2$, the hydride is at the frontier where the volume and Fe-H bonding are in balance: a small volume change has a large influence on the stability of the Fe moment as observed experimentally. Spin polarized band structure calculations on these compounds in particular $\mathrm{YFe}_{2} \mathrm{H}_{x}$ compounds would be very useful to go deeper in the interpretation.

\section{ACKNOWLEDGMENTS}

The authors are particularly grateful to I. Goncharenko, who unfortunately passed away during the accomplishment of this work. This article is dedicated to his memory. The Laboratoire Leon Brillouin and the Institut Laue Langevin are warmly acknowledged for providing the neutron facilities. The financial support of the projects 202/09/0030 and 202/09/ 531027 GA CR and that of the CNRS-ASRT cooperation program is also warmly acknowledged. The CNRS interdisciplinary energy program PR09-3.1.1.2 COREMAG is also warmly acknowledged for financial support. *olivier.isnard@grenoble.cnrs.fr

${ }^{1}$ G. Wiesinger and G. Hilscher, in Handbook of Magnetic Materials, vol. 17, edited by K. H. J. Buschow (Elsevier North-Holland, Amsterdam, 2008), pp. 293.

${ }^{2}$ I. N. Goncharenko, I. Mirebeau, A. V. Irodova, and E. Suard, Phys. Rev. B 56, 2580 (1997).

${ }^{3}$ I. N. Goncharenko, I. Mirebeau, A. V. Irodova, and E. Suard, Phys. Rev. B 59, 9324 (1999).

${ }^{4}$ P. Cadavez-Peres, I. N. Goncharenko, and I. Mirebeau, Appl. Phys. A 74, S692 (2002).

${ }^{5}$ V. Paul-Boncour, L. Guénée, M. Latroche, A. Percheron-Guégan, B. Ouladdiaf, and F. Bourée-Vigneron, J. Sol. State Chem. 142, 120 (1999).

${ }^{6}$ V. Paul-Boncour, S. M. Filipek, A. Percheron-Guégan, I. Marchuk, and J. Pielaszek, J. Alloys Compd. 317-318, 83 (2001).

${ }^{7}$ V. Paul-Boncour and A. Percheron-Guegan, J. Alloys Compd. 293295, 237 (1999).

${ }^{8}$ J. Ropka, R. Cerny, V. Paul-Boncour, and T. Proffen, J. Sol. State Chem. 182, 1907 (2009).

${ }^{9}$ T. Leblond, V. Paul-Boncour, F. Cuevas, O. Isnard, and J. F. Fernandez, Int. J. Hyd. Energ. 34, 2278 (2009).

${ }^{10}$ V. Paul-Boncour and S. Matar, Phys. Rev. B 70, 184435 (2004).

${ }^{11}$ V. Paul-Boncour, G. André, F. Bourée, M. Guillot, G. Wiesinger, and A. Percheron-Guégan, Physica B 350, e27 (2004).

${ }^{12}$ V. Paul-Boncour, M. Guillot, G. Wiesinger, and G. André, Phys. Rev. B 72, 174430 (2005)

${ }^{13}$ S. Ishida, S. Asano, and J. Ishida, J. Phys. Soc. Jap. 54, 4695 (1985).

${ }^{14}$ K. Hoshi, J. Phys. Soc. Jap. 57, 3112 (1988).

${ }^{15}$ H. Wada, M. Hada, M. Shiga, and Y. Nakamura, J. Phys. Soc. Jap. 59, 701 (1990).

${ }^{16}$ S. Asano and J. Ishida, J. Phys.: Condens. Matter 1, 8501 (1991).

${ }^{17}$ J. J. M. Franse, J. Magn. Magn. Mat. 31-34, 819 (1983).

${ }^{18}$ Y. Muraoka, M. Shiga, and Y. Nakamura, J. Magn. Magn. Mat. 31-34, 121 (1983).
${ }^{19}$ K. Hataway and J. Cullen, J. Phys. Condens. Matter 3, 8911 (1991).

${ }^{20}$ V. Paul-Boncour, M. Guillot, G. André, F. Bourée, G. Wiesinger, and A. Percheron-Guégan, J. Alloys Compd. 404-406, 355 (2005).

${ }^{21}$ V. Paul-Boncour and T. Mazet, J. Appl. Phys. 105, 013914 (2009).

${ }^{22}$ V. Paul-Boncour, L. Guénée, M. Latroche, and A. PercheronGuégan, J. Alloys Compd. 255, 195 (1997).

${ }^{23}$ T. Leblond, V. Paul-Boncour, and A. Percheron-Guégan, J. Alloys Compd. 446-447, 419 (2007).

${ }^{24}$ I. N. Goncharenko, J. M. Mignot, and I. Mirebeau, Neutron news 7, 29 (1996).

${ }^{25}$ J. Kamarad, Z. Machatova, and Z. Arnold, Rev. Sci. Instrum. 75, 5022 (2004).

${ }^{26}$ See Supplemental Material at http://link.aps.org/supplemental/ 10.1103/PhysRevB.84.094429 for neutron diffraction patterns, refinement results and details information on the $\mathrm{YFe}_{2} \mathrm{D}_{4.2}$ crystal structures in the cubic, rhombohedral and monoclinic symmetry.

${ }^{27}$ H. Sugiura, I. Marchuk, V. Paul-Boncour, A. Percheron-Guégan, T. Kitazawa, and S. M. Filipek, J. Alloys Compd. 356-357, 32 (2003).

${ }^{28}$ H. Sugiura, S. M. Filipek, V. Paul-Boncour, and I. Marchuk, Polish J. Chem. 82, 1959 (2008).

${ }^{29}$ Y. Nishihara, J. Magn. Magn. Mat. 70, 75 (1987).

${ }^{30}$ K. Irisawa, A. Fujita, K. Fukamichi, M. Yamada, H. Mitamura, T. Goto, and K. Koyama, Phys. Rev. B 70, 214405 (2004).

${ }^{31}$ D. Bagayoko and J. Callaway, Phys. Rev. B 28, 5419 (1983).

${ }^{32}$ V. L. Moruzzi, P. M. Marcus, and J. Kübler, Phys. Rev. B 39, 6957 (1989).

${ }^{33}$ W. Ludorf, M. H. Abd-Elmeguid, and H. Micklitz, J. Magn. Magn. Mat. 78, 171 (1989).

${ }^{34}$ A. Fujita, K. Fukamichi, M. Yamada, and T. Goto, Phys. Rev. B 73, 104420 (2006).

${ }^{35}$ O. Prokhnenko, C. Ritter, Z. Arnold, O. Isnard, J. Kamarad, A. Pirogov, A. Teplykh, and A. Kuchin, J. Appl. Phys. 92, 385 (2002). 
${ }^{36}$ S. Niziol, R. Zach, M. Bacmann, D. Fruchart, O. Isnard, S. Miraglia, and J. L. Soubeyroux, J. Alloys Compd. 262, 202 (1997).

${ }^{37}$ O. Isnard, R. Zach, S. Niziol, M. Bacmann, S. Miraglia, J. L. Soubeyroux, and D. Fruchart, J. Magn. Magn. Mat. 140-144, 1073 (1995).

${ }^{38}$ J. G. M. Armitage, T. Dumelow, R. H. Mitchell, P. C. Riedi, J. S. Abell, P. Mohn, and K. Schwarz, J. Phys. F: Met. Phys. 16, L141 (1986).

${ }^{39}$ J. Lyubina, K. Nenkov, L. Schultz, and O. Gutfleisch, Phys. Rev. Lett. 101, 177203 (2008).
${ }^{40}$ A. Fujita, S. Fujjeda, Y. Hasegawa, and K. Fukamichi, Phys. Rev. B 67, 104416 (2003).

${ }^{41}$ P. Rhodes and E. P. Wohlfarth, Proc. R. Soc. London, Ser. A 273, 247 (1963).

${ }^{42}$ T. Moriya, J. Magn. Magn. Mat. 14, 1 (1979).

${ }^{43}$ T. Moriya, J. Magn. Magn. Mat. 100, 261 (1991).

${ }^{44}$ M. Brouha and K. H. J. Buschow, J. Appl. Phys. 44, 1813 (1973).

${ }^{45}$ V. Paul-Boncour, J. Alloys Compd. 367, 185 (2004).

${ }^{46}$ M. Pajda, R. Ahuja, B. Johansson, J. M. Wills, H. Figiel, A. Paja, and O. Eriksson, J. Phys.: Condens. Matter 8, 3373 (1996). 